\title{
Strong spurious transcription likely contributes to DNA insert bias in typical metagenomic clone libraries
}

\author{
Kathy N. Lam and Trevor C. Charles*
}

\begin{abstract}
Background: Clone libraries provide researchers with a powerful resource to study nucleic acid from diverse sources. Metagenomic clone libraries in particular have aided in studies of microbial biodiversity and function, and allowed the mining of novel enzymes. Libraries are often constructed by cloning large inserts into cosmid or fosmid vectors. Recently, there have been reports of GC bias in fosmid metagenomic libraries, and it was speculated to be a result of fragmentation and loss of AT-rich sequences during cloning. However, evidence in the literature suggests that transcriptional activity or gene product toxicity may play a role.

Results: To explore possible mechanisms responsible for sequence bias in clone libraries, we constructed a cosmid library from a human microbiome sample and sequenced DNA from different steps during library construction: crude extract DNA, size-selected DNA, and cosmid library DNA. We confirmed a GC bias in the final cosmid library, and we provide evidence that the bias is not due to fragmentation and loss of AT-rich sequences but is likely occurring after DNA is introduced into Escherichia coli. To investigate the influence of strong constitutive transcription, we searched the sequence data for promoters and found that $r p o D / \sigma^{70}$ promoter sequences were underrepresented in the cosmid library. Furthermore, when we examined the genomes of taxa that were differentially abundant in the cosmid library relative to the original sample, we found the bias to be more correlated with the number of $r p o D / \sigma^{70}$ consensus sequences in the genome than with simple GC content.
\end{abstract}

Conclusions: The GC bias of metagenomic libraries does not appear to be due to DNA fragmentation. Rather, analysis of promoter sequences provides support for the hypothesis that strong constitutive transcription from sequences recognized as $r p o D / \sigma^{70}$ consensus-like in E. coli may lead to instability, causing loss of the plasmid or loss of the insert DNA that gives rise to the transcription. Despite widespread use of $E$. coli to propagate foreign DNA in metagenomic libraries, the effects of in vivo transcriptional activity on clone stability are not well understood. Further work is required to tease apart the effects of transcription from those of gene product toxicity.

Keywords: Metagenomics, Cosmid, Fosmid, Cloning bias, GC bias, Metagenomic libraries, E. coli host, Spurious transcription, Sigma 70

\section{Background}

Clone libraries can be generated using a range of source material, from the DNA of a single organism to the DNA from environmental sources representing often complex microbial communities. Libraries generated from microbial communities are called metagenomic libraries, and they have been central to a powerful methodology contributing to understanding the diversity of

* Correspondence: tcharles@uwaterloo.ca

Department of Biology, University of Waterloo, Waterloo, ON, Canada microbial communities, expanding the knowledge of gene function, and mining for novel sequences encoding functions of interest. These activities all fall under the umbrella of functional metagenomics and require cloning the DNA, typically using low-copy vectors such as cosmids or fosmids. Cloned DNA is typically propagated in Escherichia coli, and if the vector host range allows, the DNA can subsequently be transferred to other surrogate hosts that may be more suitable for heterologous expression.

\section{Ciomed Central}


The general assumption in cloning-based metagenomic approaches is that foreign DNA can be stably maintained in E. coli and that the cloned DNA is a fair representation of the original sample. However, it has been previously observed that fosmid libraries exhibit a GC bias [1, 2]. In general, such cloning biases may affect conclusions derived from analysis of the clone libraries. The observed GC bias of fosmid libraries was suggested to be due to fragmentation and subsequent loss of AT-rich sequences during the cloning process, purportedly because AT-rich sequences have fewer hydrogen bonds which makes them more vulnerable to non-perpendicular shear forces [1]. Other possible reasons for the bias in libraries include transcriptional activity of the cloned DNA [3] as well as toxicity from expressed genes [4, 5]. Though the exact mechanism(s) by which GC bias occurs has not yet been fully elucidated, the fragmentation explanation has been echoed by others [6, 7] despite being purely speculative and lacking experimental support. Indeed, in our experience, extracting high-molecular-weight genomic DNA from low-GC organisms is no more difficult than from E. coli. We have previously constructed genomic libraries in cosmid vectors using DNA from Bacteroides thetaiotaomicron and Bacteroides fragilis (both 43\% GC) with no difficulties obtaining high-quality DNA [8]. Furthermore, we have observed that on occasion, cosmid clones from metagenomic libraries appear to have suffered insert loss, which we discuss in greater detail in the "Results and discussion" section below. Therefore, it seemed to us that the suggestion by Temperton et al. [1] that the
GC bias in cosmid/fosmid libraries might be due to fragmentation of AT-rich sequences was unlikely to be true; rather, we believe that events occurring in vivo may be contributing substantially to the sequence bias of libraries.

We investigated the nature of this GC bias, to characterize whether, and by what mechanism, biases may be introduced into our own cosmid libraries. In particular, we wished to determine if fragmentation was a major cause of bias, or if there is evidence that the bias was indeed occurring in vivo. To answer this question, we constructed a cosmid library using DNA isolated from pooled human fecal samples, saving a portion of the DNA from three steps of the library construction process: (1) the crude extract DNA, (2) the size-selected DNA, and (3) the cloned DNA from the constructed cosmid library (Fig. 1). The DNA samples were sequenced and the resulting datasets were analyzed to investigate if, where, and how any bias may have been introduced. Consistent with the aforementioned studies, we observed GC bias in our constructed cosmid library. However, our results indicate that fragmentation of DNA does not cause any significant bias; rather, our results are consistent with the hypothesis that the bias occurs after DNA is introduced into the $E$. coli host.

\section{Results and discussion}

\section{DNA sampling and sequencing results}

We collected DNA at the three main steps of cosmid library construction: the crude extract DNA, the size-selected DNA, and the final cosmid library DNA (Fig. 1).

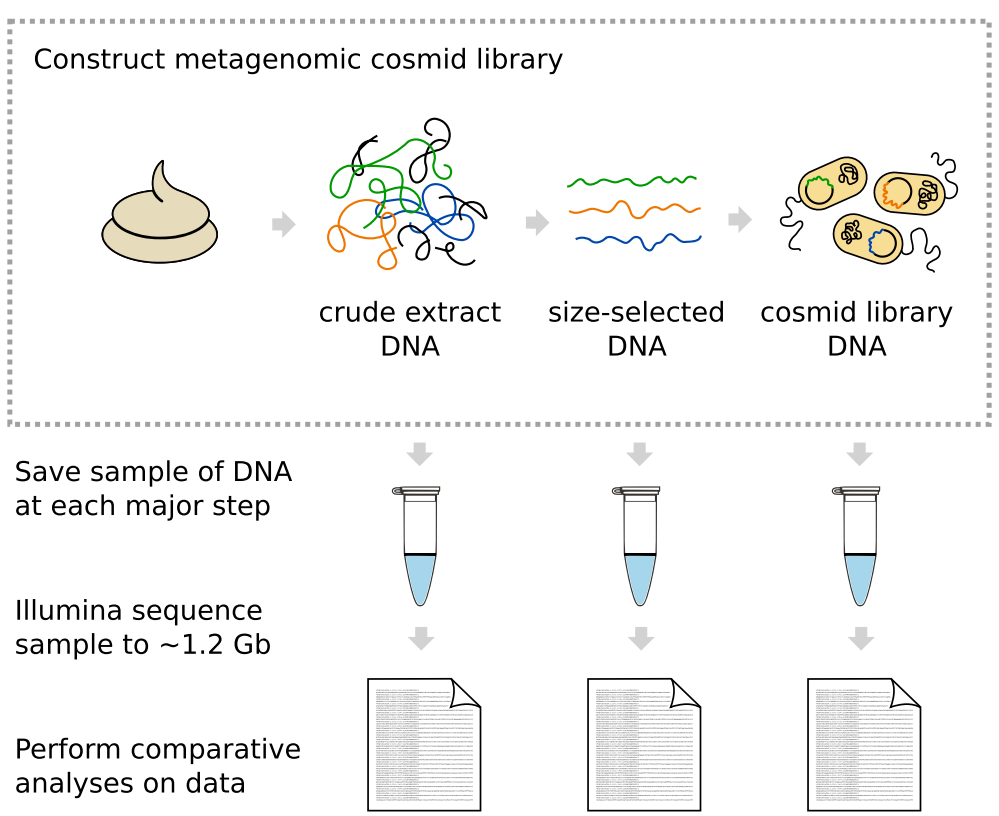

Fig. 1 Overview of the experimental design for this study. A pooled human fecal sample was used to construct a metagenomic cosmid library, during which DNA from three distinct steps was collected and sequenced in order to investigate possible sequence biases and at what steps the biases were introduced 
Before sequencing, we first checked the quality of each sample by gel electrophoresis (Fig. 2). As expected, the crude extract was the only sample that contained a heavy smear of fragmented DNA; the selection for highmolecular-weight DNA greatly reduced fragmented DNA, as evidenced by its absence from the size-selected sample. The cosmid library sample exhibited the characteristic multiple banding pattern representing the various possible conformations of uncut circular DNA.

After confirming DNA quality, the samples were paired-end sequenced on an Illumina HiSeq 2000 platform, generating $\sim 1.2 \mathrm{~Gb}$ of DNA sequence per sample. We expected that the cosmid library would be contaminated with $E$. coli genomic DNA and cosmid vector DNA as a result of (1) isolating cosmid DNA from $E$. coli cells and (2) the fact that each and every cosmid clone sequenced included its vector backbone. Thus, for fair treatment, we subtracted E. coli and pJC8 sequences from all samples (see "Methods" section). For E. coli and pJC8, respectively, 6701 and 164 reads were removed from crude extract data $(\sim 0.05 \%$ of all reads); 9273 and 2410 from size-selected data ( 0.09\%); and 851,410 and $2,130,004$ from the cosmid library DNA ( 23\%). As expected, the dataset originating from the cosmid library sample had the highest number of reads subtracted. Though the crude extract and size-selected samples contained a small amount, these likely represent true environmental sequences; however, their subtraction

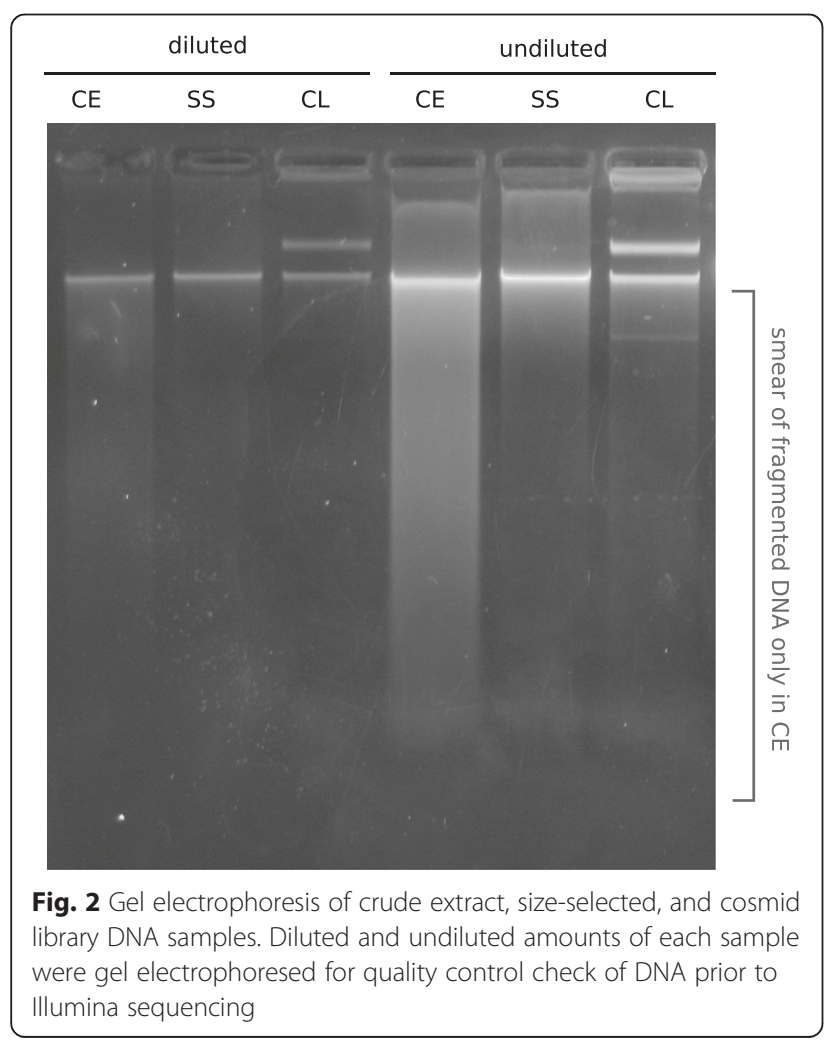

was necessary for equal treatment of all samples, and the small fraction removed should not affect overall conclusions from the data.

After host and vector sequence subtraction, we used Nonpareil [9] to estimate the overall sequencing coverage of the samples, which was $\sim 85 \%$ for the crude extract and size-selected samples and $\sim 95 \%$ for the cosmid library sample (Additional file 1: Figure S1). This relatively high sequencing coverage was sufficient for our comparative sequence analyses; for all subsequent results discussed in this paper, the forward and reverse sequencing reads for the three samples were analyzed separately.

\section{GC bias is not caused by fragmentation of AT-rich DNA during cloning}

Our experimental design was such that we could address whether the bias in our metagenomic library was due to fragmentation of DNA during cloning. Because we sequenced both crude extract and size-selected samples, we could determine whether the removed fragmented DNA from the crude extract (visible in Fig. 2) led to a bias in the size-selected DNA sample. We examined percent GC in each of the three datasets and found that the GC bias was only present in the final cosmid library and not the size-selected sample (Table 1), effectively ruling out fragmentation as the mechanism for cosmid library bias.

After confirming that the bias occurs post size selection, we next asked if certain taxa were differentially represented across the samples to see if this would point to a possible reason for library sequence bias. We used Taxy [10] as well as Taxy-Pro [11] as part of the CoMet web server [12] to do a fast preliminary comparison of taxa abundance across the three different samples. Taxy calculates $k$ mer frequencies for the dataset and then uses mixture modeling of $k$-mer frequencies of sequenced genomes to obtain a profile similar to that of the sample, whereas Taxy-Pro has a similar modeling approach but uses protein domains rather than $k$-mer frequencies. Both tools generated very similar profiles for the crude extract and the size-selected DNA but a very different profile for the cosmid library DNA (data not shown), supporting the percent

Table 1 Percent GC of crude extract, size-selected, and cosmid library datasets. GC content was calculated after subtraction of E. coli and vector DNA from all samples

\begin{tabular}{llll}
\hline Sample/dataset & No. reads & No. Mb & \% GC \\
\hline Crude extract F & $6,654,484$ & 599 & 47.7 \\
Crude extract R & $6,654,567$ & 599 & 47.8 \\
Size-selected F & $6,645,306$ & 598 & 46.9 \\
Size-selected R & $6,645,817$ & 598 & 46.9 \\
Cosmid library F & $5,134,020$ & 462 & 53.0 \\
Cosmid library R & $5,191,538$ & 467 & 53.1 \\
\hline
\end{tabular}


GC results. With positive results from this preliminary work, we then performed more thorough taxonomic analyses using two different approaches; in the first, all sequencing reads were used, and in the second, only the $16 \mathrm{~S}$ rRNA gene-containing reads were used (see "Methods" section).

In the first approach, we used the Metagenome Phylogenetic Analysis (MetaPhlAn) tool, a profiling tool that maps reads against clade-specific marker sequences [13] to estimate sample composition down to the species level (Additional file 2: Table S1). We examined the abundance of the top four most common phyla in human gut metagenomes to see whether there were large overall changes in taxa abundance across the samples (Fig. 3). The crude extract and sizeselected samples showed high Firmicutes and Bacteroidetes content with lower levels of Actinobacteria and Proteobacteria, compositions that are typical of gut-derived samples [14-16]. Notably, our results indicated that the cosmid library sample underwent a substantial decrease in the Firmicutes, accompanied by a comparably substantial increase in the Actinobacteria. These results were consistent with the percent GC analysis, as members of the Firmicutes phylum are generally known to be low-GC, and those of the Actinobacteria, high-GC. We also examined the MetaPhlAn results at the species level to see which genomes may be under- or overrepresented in the cosmid library, choosing to examine the top 50 most differentially abundant species (Fig. 4). Several members of the Bifidobacterium genus were substantially overrepresented in the cosmid library while many members of the Firmicutes were completely or very nearly lost; for example, Eubacterium rectale, Ruminococcus bromii, and Faecalibacterium prausnitzii were all highly abundant in the original sample.
In our second approach, we identified reads in the datasets that were from the 16S rRNA gene, and used the RDP classifier to classify these to the genus level (Additional file 3: Figure S2). We observed that analyses using only $16 \mathrm{~S}$ rRNA gene-containing reads showed high agreement with analyses carried out using all reads (i.e., Fig. 4), indicating that $16 \mathrm{~S}$ rRNA gene content tracks well with genomic content in large-insert cosmid libraries. Both of our approaches provided similar results, and both were in agreement with percent GC, Taxy, and Taxy-Pro results, all of which provide compelling evidence that cosmid library biases are not due to fragmentation of AT-rich sequences during the cloning process.

\section{GC content may be merely a proxy for $E$. coli constitutive promoter content}

From these results, our own previous experiences, and what was previously known in the literature, we had reason to suspect that the cause of the bias occurred in vivo. We are not the first to suggest that sequences from AT-rich genomes may resemble the constitutive $E$. coli promoter $[17,18]$, particularly the -10 Pribnow box. To investigate whether transcription of the insert may be having a negative effect on its maintenance by the host cell, we analyzed the sequence data from the three samples for $E$. coli consensus promoter sequences; in particular, we were interested in examining the data for differential abundance of the $r p o D / \sigma^{70}$ consensus sequence, as $\sigma^{70}$ is the "house-keeping" sigma factor whose promoters are constitutive.

We used the known promoter consensus sequence for $r p o D / \sigma^{70}$ [19], and, as negative controls, we used the consensus sequence for: $r p o E / \sigma^{24}[20] ; r p o H / \sigma^{32}$ [21]; rpoN $/ \sigma^{54}$, which has a GC-rich consensus [22]; as well as the primary sigma factor of Bacteroides species, $\sigma^{\mathrm{ABfr}}$ [23], because the Bacteroides genus had comparable

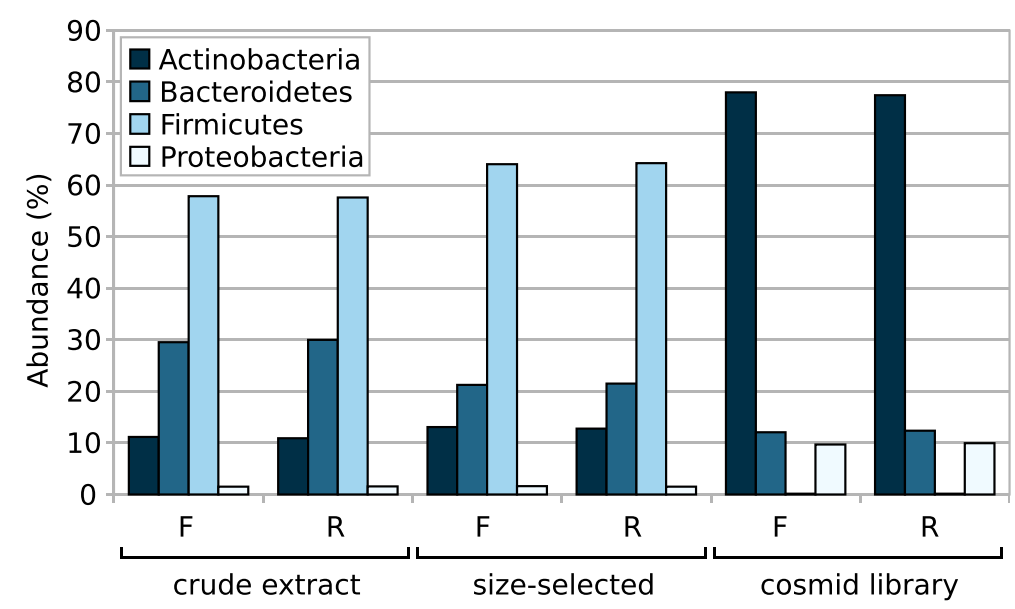

Fig. 3 Histogram of abundance of the top four phyla in crude extract, size-selected, and cosmid library samples. Abundance of the Actinobacteria, Bacteroidetes, Firmicutes, and Proteobacteria phyla in each sample, as determined using MetaPhIAn 


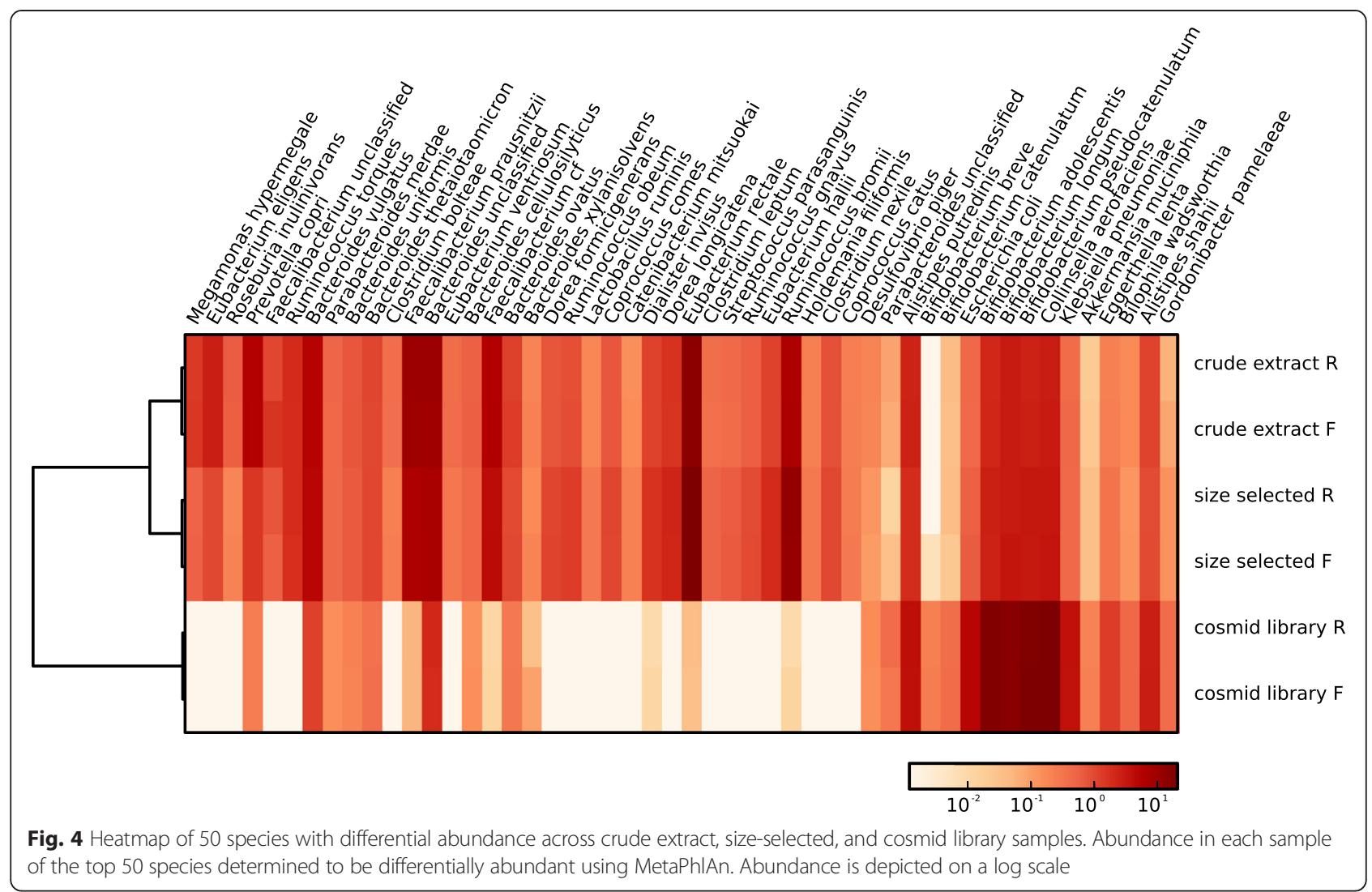

abundance across the three samples (Additional file 3: Figure S2) and because Bacteroides constitutive promoters are not recognized by $E$. coli [24]. We examined each of the three samples for relative abundance of these five consensus sequences (see "Methods" section for details). Our results showed that while the crude extract and size-selected samples had similar promoter content profiles, the cosmid library exhibited a deviation (Fig. 5). Supporting our hypothesis, only the rpoD consensus content was considerably different in abundance, by about an order of magnitude when compared to either the crude extract or size-selected sample. The loss of

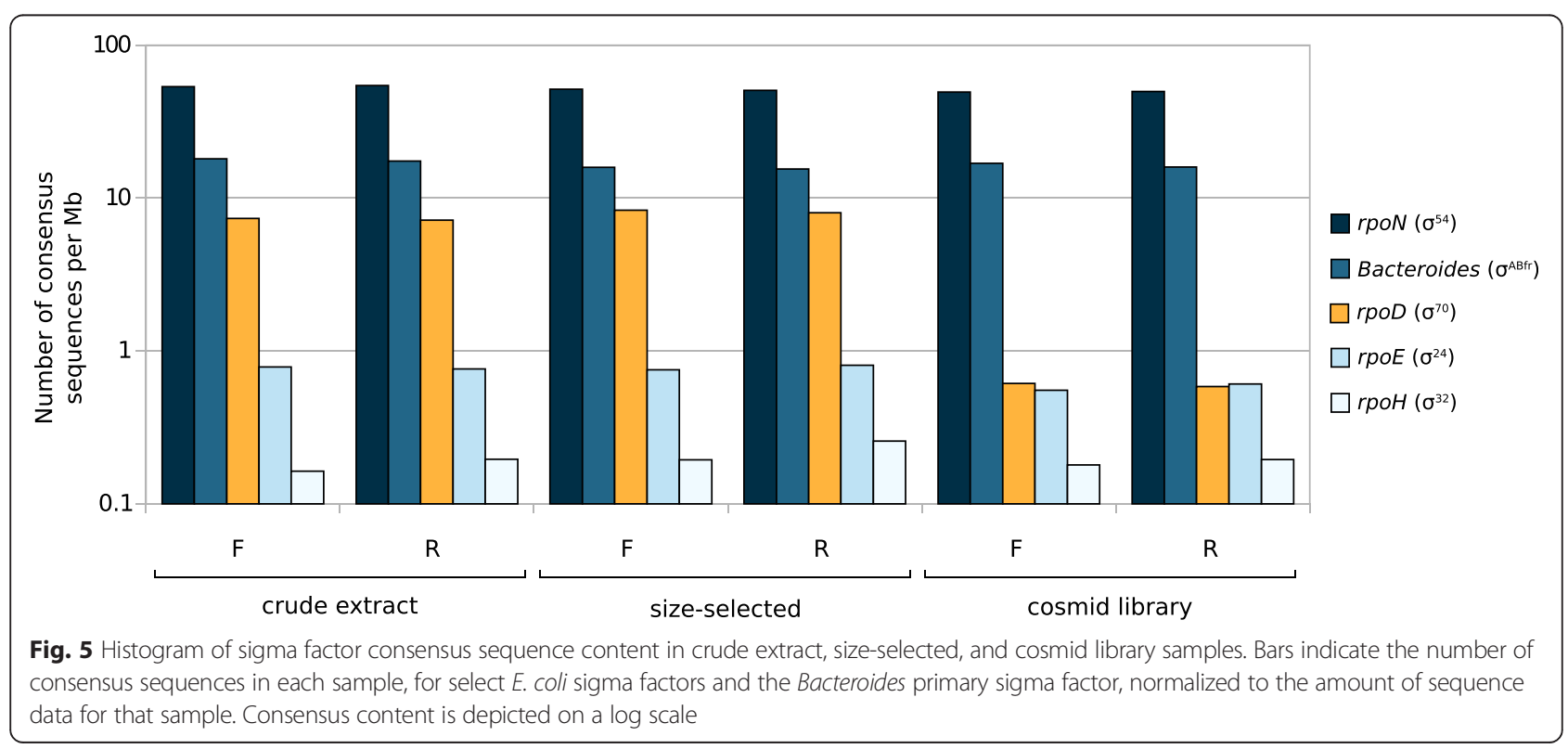


these specific sequences from the cosmid library suggests that the widely used cloning host $E$. coli may be problematic for cosmid-cloned fragments of DNA that incidentally contain constitutively active rpoD sequences; indeed, these findings are supported by previous reports in the literature, which we discuss in more detail in the following section. If E. coli does in fact exclude constitutively active $r p o D$-containing sequences, simply switching to a different cloning/library host (even if it were possible) would likely alleviate one problem only to introduce another, as all organisms have sequences from which constitutive transcription arises. It may be that multiple backgrounds, with different constitutively active sequences, are required for the maintenance of metagenomic libraries in an effort to increase sample representativeness.

Given that rpoD promoter sequences were underrepresented in the cosmid library and that certain species appear to be over- or underrepresented, we next asked whether a species' abundance in the cosmid library could be predicted from the rpoD consensus content of its genome. And in particular, is rpoD consensus content more predictive of library abundance than GC content? To answer our questions, we turned to the results of our MetaPhlAn analysis, which gave us a list of the top 50 most differentially abundant species (Fig. 4). To analyze the genomes of the species for possible sequence determinants of library abundance, we used the NCBI genome database to find sequenced representatives of each species where possible and retrieved 46 genomes (complete, draft, or whole genome shotgun sequences; see "Methods" section for details); for each genome, we calculated the percent GC as well as the number of rpoD consensus promoter sequences present (Additional file 4: Table S2). Next, to quantify bias in the cosmid library relative to the original sample (the crude extract), we calculated the change in abundance of the 46 species (using the average abundance of the forward and reverse datasets). We then plotted the change in abundance first against genome percent GC (Fig. 6a) and second against rpoD consensus content, normalizing to genome size (Fig. 6b). Our results show that while library bias only generally correlates with GC content, library bias correlates surprisingly well with the rpoD consensus content of the genome.

These results suggest that GC content may be only a rough proxy for rpoD consensus content (as rpoD consensus sequences are AT-rich), but GC content itself may not be an accurate predictor of library presence/ abundance; indeed, in some cases, a genome may have a moderate or relatively high percent GC but also possess an unusually high rpoD consensus content, leading to an underrepresentation in the cosmid library that could not

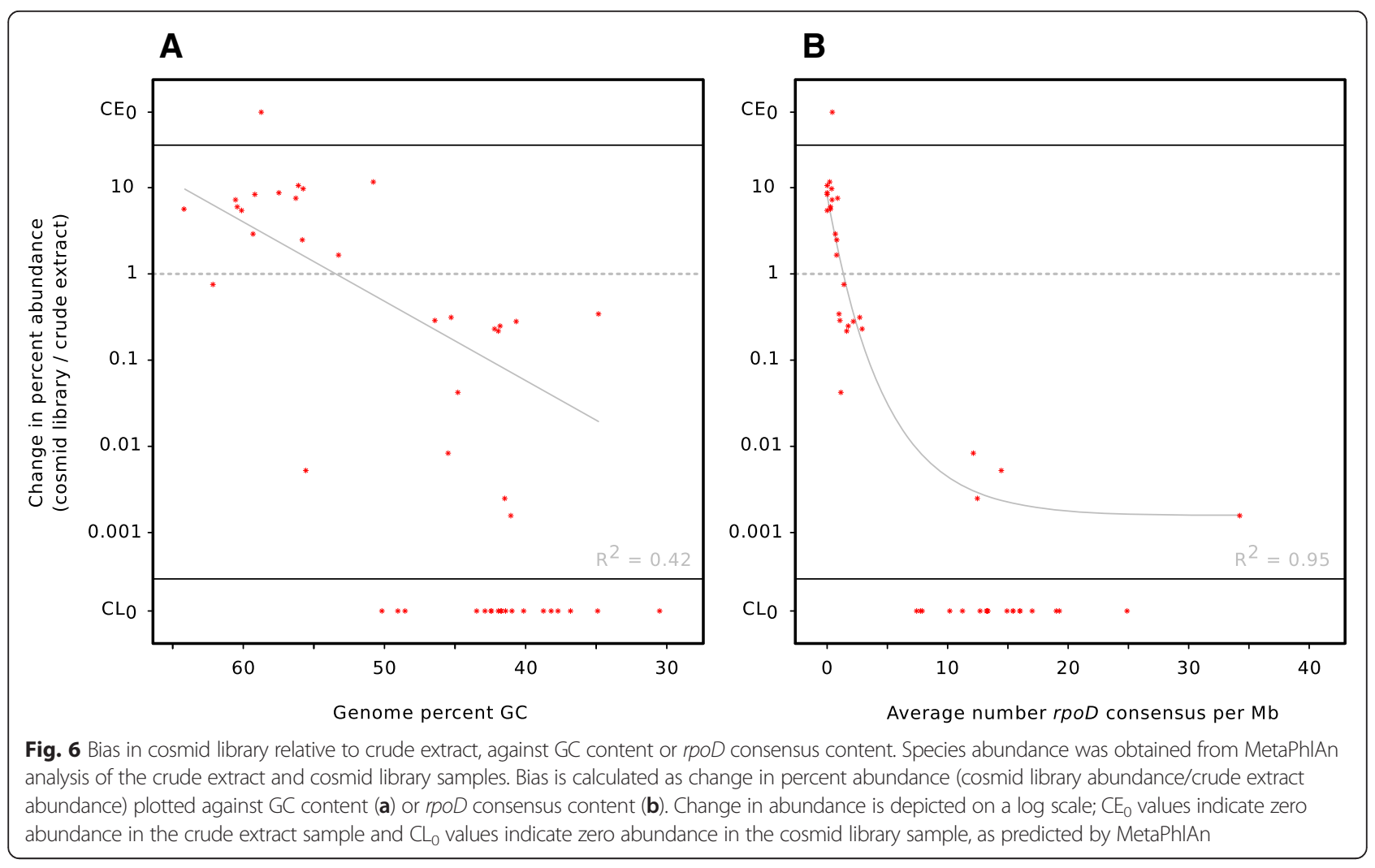


have been predicted from GC content alone (Fig. 6). In our view, these results are also consistent with the previous observation that library bias was more obvious among organisms with low GC content [2] because AT-rich genomes would have an increased number of $r p o D$ promoter-like sequences simply by chance [25].

\section{Examining the published literature: evidence for transcriptional activity of cloned AT-rich DNA interfering with stability of circular vectors}

In this report, we have presented analysis concerning metagenomic DNA. However, if rpoD consensus-like sequences are interfering with the maintenance of foreign DNA in E. coli, then the scope of the problem extends beyond metagenomics applications. Curious about the extent of the problem, we performed literature searches to find reports of experienced difficulties cloning ATrich DNA and/or investigations of possible mechanisms for those difficulties. Our search was fruitful, leading us to literature that spans the past three decades.

It was reported that there are difficulties associated with cosmid cloning of very AT-rich genomic DNA [26, 27], and even when genomic libraries can be constructed, cosmid clones may be unstable [28-31], which simply means that foreign DNA fragments are not able to be maintained in the $E$. coli library host. Thus, if selection is applied for a marker present on the vector, then in vivo events may lead to insert deletion, which has been observed by us as well as others, despite using a host that is a recA mutant [31]. This is particularly evident when the library is constructed using a high-copy number vector (e.g., one containing a ColE1-type origin of replication), which has been experienced by us and others [32] and is in agreement with the observation that F-based, single-copy fosmids perform better than multi-copy cosmids at stably maintaining insert DNA [33]. Loss of cloned sequence is even more widespread for inserts that have repetitive DNA sequences [34], as such sequences may be conducive to recombination. One way to combat insert loss is by minimizing outgrowth of the library-containing cells as much as possible [31], though this is not always feasible for shared cosmid libraries such as our Canadian MetaMicroBiome Library collection [35].

But what is the mechanism for plasmid instability? It was previously shown that transcriptional activity from a cloned strong promoter could affect plasmid stability by (1) interfering with the origin of replication via transcriptional read-through into the vector as well as (2) changing the abundance of protein products involved in plasmid copy number. Furthermore, plasmid instability was alleviated by placing transcriptional terminator sequences that flank the multiple cloning site [36]. It was also observed that strong phage promoters could only be cloned into plasmids that possess a downstream termination signal [37, 38]. Similarly, AT-rich pneumococcal DNA was found to contain a high incidence of E. coli strong promoter sequences, and that cloning of the DNA was improved by using a vector with efficient transcriptional terminators $[3,32,39]$, although analysis of a set of pneumococcal promoter-containing sequences indicated that transcription strong enough to interfere with plasmid stability may be relatively rare and that other factors could be contributing to cloning difficulty [40].

Another consideration is that efficient transcription of poly-dT (as well as poly-dG) DNA tracts may cause the DNA to form a stable complex with its own accumulated transcription products, leading to transcriptional stalling that may interfere with the replication fork [41-43]. One particularly interesting observation that has surprisingly not attracted more interest is that linear cloning vectors with transcriptional terminators provide even more stability than circular vectors with transcriptional terminators $[26,44]$. The advantage of these vectors is due to their linear conformation, but intriguingly, the mechanism remains unclear, although DNA supercoiling of plasmids is thought to play a role (Ronald Godiska, personal communication). These findings along with the aforementioned facts suggest that multiple, distinct mechanisms may be at play to cause cloning bias in E. coli, but that there is evidence that transcriptional activity of cloned DNA may be contributing to the sequence bias observed in metagenomic libraries. It is often assumed that toxicity of gene products may influence the stable maintenance or "clonability" of DNA in $E$. coli $[4,5,45]$, but it is currently unclear whether gene product toxicity is a major factor in the bias of typical clone libraries constructed using circular vectors. It is interesting to consider that cloning bias could be due primarily to purely transcriptional activity rather than the often-blamed protein toxicity.

\section{Conclusions}

The results presented in this report and what was already known from the literature together support the hypothesis that GC bias in typical clone libraries (that is, using circular vectors) is related to constitutive promoter activity of the insert in E. coli, although DNA topology as well as toxic protein effects may also influence insert and plasmid maintenance. In our analyses, we have focused only on would-be strong constitutive promoters in E. coli ( $\sigma^{70} / r p o D$ consensus sequences) because there is evidence that high-level transcription may have negative effects. It is important to acknowledge, however, that functional metagenomic approaches rely on E. coli (or other hosts) being able to transcribe and translate foreign DNA, in order to identify fragments encoding functions of interest. This ability of $E$. coli to initiate low-level transcription from diverse sources [46] and to be able to produce foreign 
proteins has been immensely advantageous for functional metagenomics and likely has contributed to the general assumption that E. coli is tolerant of foreign DNA, whether it expresses it or not. Our work, however, suggests that more careful consideration of cloning strategies may be required.

Currently, there are three outstanding questions: (1) to what extent does transcription contribute to metagenomic library bias, (2) what factors affect whether transcription will be problematic, and (3) how can transcriptional effects be minimized so that DNA can be faithfully maintained in E. coli. An important consideration may be the likelihood of an rpoD consensus sequence being cloned on any given fragment from a genome or metagenome. As an example, let us consider Ruminococcus bromii, which was one of the most highly abundant species in the original sample but became nearly absent in the cosmid library according to our analyses ( 7 versus $\sim 0.01 \%$, respectively; see Additional file 2: Table S1). R. bromii has a genome size of $2.25 \mathrm{Mb}$; theoretically, its genome can be represented in $\sim 80$ fragments if we consider that the average fragment in the particular cosmid library discussed here is $\sim 28 \mathrm{~kb}$ (data not shown). Given that there were 77 rpoD consensus sequences identified in its genome (Additional file 4: Table S2), potentially many fragments could include a sequence that behaves as a strong, constitutive promoter in E. coli. We acknowledge that although our work supports the hypothesis that constitutive transcription contributes to library bias, more concrete evidence is required to confirm this hypothesis.

If strong transcription from the insert into the vector backbone contributes in part to the observed cloning bias-affecting the origin of replication, for example-it may be helpful to use vectors that include transcriptional terminators flanking the cloning site. We are currently investigating the extent to which transcriptional terminators alleviate the cosmid library sequence bias, which may help tease apart the issue of transcription from that of gene product toxicity. While it is generally recognized that different expression hosts are needed for functional screening [45, 47-52], it is not as widely acknowledged that using $E$. coli as the sole cloning host for metagenomic DNA itself may be quite limiting due to the potential lack of sample representativeness from the outset. It is interesting that despite decades of using E. coli as "the workhorse of molecular biology," there is still much left to discover about how it tolerates exogenous DNA, which should serve as a reminder to us of how necessary it is to continually re-evaluate even our most basic methodological assumptions, particularly when they concern the inner workings of the cell.

\section{Methods}

\section{Sampling of DNA during steps of metagenomic cosmid} library construction

Methods for the construction of cosmid libraries, including the specific human gut metagenomic library discussed here (NCBI BioSample ID SAMN02324081), have been previously described in detail [8]. Briefly, DNA was extracted from pooled human fecal samples using freeze-grinding with liquid nitrogen followed by gentle lysis. Crude-extracted DNA was then size-selected by pulsed field gel electrophoresis using a CHEF Mapper Pulsed Field Gel Electrophoresis System (Bio-Rad), followed by electroelution, retaining fragments between approximately 40 and $70 \mathrm{~kb}$. The size-selected DNA was end-repaired, purified, and ligated into the Eco72I site of linearized dephosphorylated pJC8 vector DNA (Genbank accession KC149513). The ligation product was packaged into lambda phage heads using Gigapack III XL Packaging Extract (Stratagene), followed by transduction of E. coli HB101. Transductants were recovered on LB agar supplemented with tetracycline $(20 \mu \mathrm{g} / \mathrm{ml})$ and incubated overnight at $37^{\circ} \mathrm{C}$. Resulting colonies were enumerated to estimate library size ( 42,000 clones), and colonies were resuspended, pooled, and frozen at $-80^{\circ} \mathrm{C}$ to form the cosmid library stock.

During construction of the cosmid library, DNA was sampled from three steps: (1) the crude extract DNA, (2) the size-selected DNA, and (3) the final cosmid library DNA, prepared from the frozen stock using a GeneJET Plasmid Miniprep Kit (Thermo Scientific).

\section{Purification, quantification, and Illumina sequencing of DNA}

Two of the three DNA samples, the cosmid library DNA and the size-selected DNA, were sufficiently pure for Illumina sequencing, as gaged by $260 / 280$ and $260 / 230$ $\mathrm{nm}$ ratios (Nanodrop ND-1000 Spectrophotometer); however, the crude extract DNA required further purification. Crude extract DNA concentration was estimated by gel electrophoresis, using bacteriophage lambda DNA as a standard; $\sim 150 \mu \mathrm{g}$ in $1 \mathrm{ml}$ was purified and concentrated on the synchronous coefficient of drag alteration (SCODA) instrument (Boreal Genomics), using an established protocol [53].

All samples were re-quantified by gel electrophoresis, using bacteriophage lambda DNA as a standard, and $>2 \mu \mathrm{g}$ of each sample was sent to the Beijing Genomics Institute (BGI, Hong Kong) for 90-base paired-end sequencing on the Illumina HiSeq 2000 platform, using their in-house protocols and reagents for $350 \mathrm{bp}$ fragment library construction. Approximately 6.7 million reads were obtained in both the forward and the reverse direction, generating $\sim 1.2 \mathrm{~Gb}$ of sequence data per sample. All sequence data have been made publicly available (see "Data" section). 


\section{Subtraction of $E$. coli genome and cosmid vector contamination}

The cosmid library sequence data were expected to have substantial contamination with $E$. coli genomic DNA and $\mathrm{pJC} 8$ vector sequences. Sequence data were cleaned of contaminating $E$. coli genomic DNA and vector DNA, using BLAT [54] with a conservative criterion of $100 \%$ identity. To remove $E$. coli contamination, we used the genome of $E$. coli K12 MG1655 (Genbank accession U00096.3), which to our knowledge is currently the closest sequenced relative of HB101, the library host strain. To remove vector contamination, we used the sequence of pJC8 (Genbank accession KC149513), formatted to simulate Eco72I-cut, cloning-ready vector by removing the $0.8-\mathrm{kb}$ gentamicin resistance gene stuffer present between the two Eco72I sites.

\section{Taxonomic analysis}

To examine taxonomy based on only the $16 \mathrm{~S}$ rRNA gene sequences present in the data, we identified 16Scontaining reads using Infernal version 1.1 [55] and classified them using the RDP Classifier version 2.8 [56]. The classifier output was visualized using the MEtaGenome ANalyzer (MEGAN) version 5.6 [57]. To examine taxonomy using all sequence reads (i.e., not only those identified as $16 \mathrm{~S}$ reads), we used the MetaPhlAn tool version 2.0, along with its built-in scripts for visualization [13].

\section{Promoter analysis}

To estimate promoter content in the data, we searched for known sigma factor consensus sequences for the $E$. coli sigma factors, $r p o D / \sigma^{70}$ (TTGACAN $_{15-19}$ TATAAT), $r p o E / \sigma^{24}$ (GGAACTTN ${ }_{15-19}$ TCAAA), $r p o H / \sigma^{32}$ (TTG $\left.[\mathrm{A} / \mathrm{T}][\mathrm{A} / \mathrm{T}][\mathrm{A} / \mathrm{T}] \mathrm{N}_{13-14} \mathrm{CCCCAT}[\mathrm{A} / \mathrm{T}] \mathrm{T}\right), \operatorname{rpoN} / \sigma^{54}$ (TG $\left.\mathrm{GCAN}_{7} \mathrm{TGC}\right)$, as well as for the Bacteroides primary sigma factor, $\sigma^{\mathrm{ABfr}}\left(\mathrm{TTTGN}_{19-21} \mathrm{TAN}_{2}\right.$ TTTG). To do this, we used regular expression pattern matching with Python version 2.7.3; consensus promoter sequences, literature references, and regular expressions are provided (Additional file 5: Table S3).

\section{Analysis of reference genomes}

Genome sequences were downloaded from the NCBI Genbank database as complete genomes, draft genomes, or from whole genome shotgun sequencing projects. Organism names and accession numbers, as well as other relevant information, are provided (Additional file 6: Table S4).

\section{Data}

Raw Illumina sequence data are available at the NCBI Sequence Read Archive under Study SRP031898. Accession numbers for SRA Experiments are: NCBI:SRX683591 for the crude extract, NCBI:SRX683589 for the size-selected, and NCBI:SRX683586 for the cosmid library. In addition, raw data and other relevant data for this study may be accessed online through our website [58].

\section{Additional files}

Additional file 1: Figure S1. Estimate of sample sequencing coverage using Nonpareil.

Additional file 2: Table S1. Taxa abundance output from MetaPhIAn for both forward and reverse datasets of each sample.

Additional file 3: Figure S2. 165 rRNA analysis results using Infernal for identification of 165 -containing reads, RDP classifier to classify reads, and MEGAN for visualization of results.

Additional file 4: Table S2 Length, GC content, and rpoD consensus content of the 46 genomes selected for analysis.

Additional file 5: Table S3. Consensus sequences for the five sigma factors used, PMID number for the literature reference, and corresponding regular expressions used to search sequence data.

Additional file 6: Table S4. NCBI Genbank accession numbers for genome sequences of the 46 species selected for GC content and rpoD consensus content analysis.

\section{Abbreviations}

CE: crude extract; SS: size-selected; CL: cosmid library; F: forward reads; $\mathrm{R}$ : reverse reads.

\section{Competing interests}

The authors declare that they have no competing interests.

\section{Authors' contributions}

TCC and KNL designed the experiments. KNL performed the experiments, structured and performed the data analysis, and wrote the paper. TCC provided constructive criticism, revised the manuscript, and provided reagents and materials. All authors read and approved the final manuscript.

\section{Acknowledgements}

We thank the seven anonymous individuals who donated fecal samples for the construction of the CLGM1 cosmid library. We are grateful to the Shared Hierarchical Academic Research Computing Network (SHARCNET:

www.sharcnet.ca) and Compute/Calcul Canada for computing resources. Research funding was provided by a Strategic Projects Grant (381646-09) from the Natural Sciences and Engineering Research Council of Canada, by Genome Canada for the project "Microbial Genomics for Biofuels and Co-Products from Biorefining Processes" $\left(\mathrm{MGCB}^{2}\right)$, and by a University of Waterloo CIHR Research Incentive Fund. KNL was supported by a CGS-D scholarship from the Canadian Institutes of Health Research.

Received: 9 December 2014 Accepted: 1 May 2015 Published online: 20 May 2015

\section{References}

1. Temperton B, Field D, Oliver A, Tiwari B, Mühling M, Joint I, et al. Bias in assessments of marine microbial biodiversity in fosmid libraries as evaluated by pyrosequencing. ISME J. 2009;3:792-6.

2. Danhorn T, Young CR, DeLong EF. Comparison of large-insert, small-insert and pyrosequencing libraries for metagenomic analysis. ISME J. 2012;1-11.

3. Chen J-D, Morrison DA. Construction and properties of a new insertion vector, pJDC9, that is protected by transcriptional terminators and useful for cloning of DNA from Streptococcus pneumoniae. Gene. 1988;64:155-64.

4. Sorek R, Zhu Y, Creevey CJ, Francino MP, Bork P, Rubin EM. Genome-wide experimental determination of barriers to horizontal gene transfer. Science. 2007;318:1449-52.

5. Feingersch R, Béjà O. Bias in assessments of marine SAR11 biodiversity in environmental fosmid and BAC libraries? ISME J. 2009;3:1117-9. 
6. Lombard N, Prestat E, van Elsas JD, Simonet P. Soil-specific limitations for access and analysis of soil microbial communities by metagenomics. FEMS Microbiol Ecol. 2011;78:31-49.

7. Gori F, Tringe SG, Folino G, van Hijum SAFT, Op den Camp HJM, Jetten MSM, et al. Differences in sequencing technologies improve the retrieval of anammox bacterial genome from metagenomes. BMC Genomics. 2013;14:1-9

8. Lam KN, Hall MW, Engel K, Vey G, Cheng J, Neufeld JD, et al. Evaluation of a pooled strategy for high-throughput sequencing of cosmid clones from metagenomic libraries. PLoS One. 2014;9, e98968.

9. Rodriguez-R LM, Konstantinidis KT. Nonpareil: a redundancy-based approach to assess the level of coverage in metagenomic datasets. Bioinformatics. 2014:30:629-35.

10. Meinicke $P, A ß h a u e r ~ K P$, Lingner T. Mixture models for analysis of the taxonomic composition of metagenomes. Bioinformatics. 2011;27:1618-24.

11. Klingenberg $H$, Aßhauer KP, Lingner T, Meinicke P. Protein signature-based estimation of metagenomic abundances including all domains of life and viruses. Bioinformatics. 2013;29:973-80.

12. Lingner T, Aßhauer KP, Schreiber F, Meinicke P. CoMet-a web server for comparative functional profiling of metagenomes. Nucleic Acids Res. 2011;39:W518-23.

13. Segata N, Waldron L, Ballarini A, Narasimhan V, Jousson O, Huttenhower C. Metagenomic microbial community profiling using unique clade-specific marker genes. Nat Methods. 2012;9:811-4.

14. El Kaoutari A, Armougom F, Gordon Jl, Raoult D, Henrissat B. The abundance and variety of carbohydrate-active enzymes in the human gut microbiota. Nat Rev Microbiol. 2013;11:497-504.

15. Spor A, Koren O, Ley R. Unravelling the effects of the environment and host genotype on the gut microbiome. Nat Rev Microbiol. 2011;9:279-90.

16. Marchesi JR. Human distal gut microbiome. Environ Microbiol. 2011:13:3088-102

17. Djordjevic G, Bojovic B, Banina A, Topisirovic L. Cloning of promoter-like sequences from Lactobacillus paracasei subsp. paracasei CG11 and their expression in Escherichia coli, Lactococcus lactis, and Lactobacillus reuteri. Can J Microbiol. 1994:40:1043-50

18. Morrison DA, Jaurin B. Streptococcus pneumoniae possesses canonical Escherichia coli (sigma 70) promoters. Mol Microbiol. 1990:4:1143-52.

19. Shimada T, Yamazaki Y, Tanaka K, Ishihama A. The whole set of constitutive promoters recognized by RNA polymerase RpoD holoenzyme of Escherichia coli. PLoS One. 2014;9, e90447.

20. Rhodius VA, Suh WC, Nonaka G, West J, Gross CA. Conserved and variable functions of the sigmaE stress response in related genomes. PLoS Biol. 2006:4, e2.

21. Nonaka G, Blankschien M, Herman C, Gross CA, Rhodius VA. Regulon and promoter analysis of the $E$. coli heat-shock factor, sigma32, reveals a multifaceted cellular response to heat stress. Genes Dev. 2006:20:1776-89.

22. Zhao K, Liu M, Burgess RR. Promoter and regulon analysis of nitrogen assimilation factor, sigma54, reveal alternative strategy for E. coli MG1655 flagellar biosynthesis. Nucleic Acids Res. 2010;38:1273-83.

23. Bayley DP, Rocha ER, Smith CJ. Analysis of cepA and other Bacteroides fragilis genes reveals a unique promoter structure. FEMS Microbiol Lett. 2000:193:149-54.

24. Mastropaolo MD, Thorson ML, Stevens AM. Comparison of Bacteroides thetaiotaomicron and Escherichia coli 16S rRNA gene expression signals. Microbiology. 2009;155:2683-93.

25. Mulligan ME, McClure WR. Analysis of the occurrence of promoter-sites in DNA Nucleic Acids Res. 1986:14:109-26.

26. Godiska R, Mead D, Dhodda V, Wu C, Hochstein R, Karsi A, et al. Linear plasmid vector for cloning of repetitive or unstable sequences in Escherichia coli. Nucleic Acids Res. 2010;38, e88.

27. Gardner MJ, Hall N, Fung E, White O, Berriman M, Hyman RW, et al. Genome sequence of the human malaria parasite Plasmodium falciparum. Nature. 2002:419:498-511.

28. Hattori Y, Omori H, Hanyu M, Kaseda N, Mishima E, Kaneko T, et al. Ordered cosmid library of the mesorhizobium loti MAFF303099 genome for systematic gene disruption and complementation analysis. Plant Cell Physiol. 2002:43:1542-57.

29. Brayton KA, De Villiers EP, Fehrsen J, Nxomani C, Collins NE, Allsopp BA. Cowdria ruminantium DNA is unstable in a SuperCos1 library. Onderstepoort J Vet Res. 1999;117:111-7.
30. Rhee D-K. Instability of pneumococcus library in pHC79 and pACYC184. Arch Pharm Res. 1995:18:31-7.

31. Shoemaker NB, Barber RD, Salyers AA. Cloning and characterization of a Bacteroides conjugal tetracycline-erythromycin resistance element by using a shuttle cosmid vector. J Bacteriol. 1989;171:1294-302.

32. Chen J-D, Morrison DA. Cloning of Streptococcus pneumoniae DNA fragments in Escherichia coli requires vectors protected by strong transcriptional terminators. Gene. 1987:55:179-87.

33. Kim U-J, Shizuya H, de Jong PJ, Birren B, Simon MI. Stable propagation of cosmid sized human DNA inserts in an $\mathrm{F}$ factor based vector. Nucleic Acids Res. 1992;20:1083-5.

34. Case ST. Selective deletion of large segments of Balbiani ring DNA during molecular cloning. Gene. 1982;20:169-76.

35. Neufeld JD, Engel K, Cheng J, Moreno-Hagelsieb G, Rose DR, Charles TC. Open resource metagenomics: a model for sharing metagenomic libraries. Stand Genomic Sci. 2011:5:1-12.

36. Stueber D, Bujard $\mathrm{H}$. Transcription from efficient promoters can interfere with plasmid replication and diminish expression of plasmid specified genes. EMBO J. 1982:1:1399-404.

37. Gentz R, Langner A, Chang ACY, Cohent SN, Bujard H. Cloning and analysis of strong promoters is made possible by the downstream placement of a RNA termination signal. Proc Natl Acad Sci. 1981;78:4936-40.

38. Kwon Y-S, Kim J, Kang C. Viability of E. coli cells containing phage RNA polymerase and promoter: interference of plasmid replication by transcription. Genet Anal Biomol Eng. 1998;14:133-9.

39. Stassi DL, Lacks SA. Effect of strong promoters on the cloning in Escherichia coli of DNA fragments from Streptococcus pneumoniae. Gene. 1982;18:319-28

40. Dillard JP, Yother J. Analysis of Streptococcus pneumoniae sequences cloned into Escherichia coli: effect of promoter strength and transcription terminators. J Bacteriol. 1991;173:5105-9.

41. Kiyama R, Oishi M. Instability of plasmid DNA maintenance caused by transcription of poly (dT)-containing sequences in Escherichia coli. Gene. 1994;150:57-61.

42. Kiyama R, Oishi M. In vitro transcription of a poly (dA) x poly ( $d T$ )-containing sequence is inhibited by interaction between the template and its transcripts. Nucleic Acids Res. 1996;24:4577-83.

43. Krasilnikova MM, Samadashwily GM, Krasilnikov AS, Mirkin SM. Transcription through a simple DNA repeat blocks replication elongation. EMBO J. 1998;17:5095-102

44. Godiska R, Patterson M, Schoenfeld T, Mead DA. Beyond pUC: vectors for cloning unstable DNA. In: Kieleczawa J, editor. Optim DNA Seq Process. Sudbury, Massachusetts: Jones and Bartlett Publishers; 2005. p. 55-75.

45. Taupp M, Mewis K, Hallam SJ. The art and design of functional metagenomic screens. Curr Opin Biotechnol. 2011:22:1-8.

46. Miller WG, Simons RW. DNA from diverse sources manifests cryptic low-level transcription in Escherichia coli. Mol Microbiol. 1990:4:881-93.

47. Craig JW, Chang F-Y, Kim JH, Obiajulu SC, Brady SF. Expanding smallmolecule functional metagenomics through parallel screening of broadhost-range cosmid environmental DNA libraries in diverse proteobacteria. Appl Environ Microbiol. 2010;76:1633-41.

48. Aakvik T, Degnes KF, Dahlsrud R, Schmidt F, Dam R, Yu L, et al. A plasmid RK2-based broad-host-range cloning vector useful for transfer of metagenomic libraries to a variety of bacterial species. FEMS Microbiol Lett. 2009;296:149-58.

49. Li Y, Wexler M, Richardson DJ, Bond PL, Johnston AWB. Screening a wide host-range, waste-water metagenomic library in tryptophan auxotrophs of Rhizobium leguminosarum and of Escherichia coli reveals different classes of cloned trp genes. Environ Microbiol. 2005;7:1927-36.

50. Cheng J, Pinnell L, Engel K, Neufeld JD, Charles TC. Versatile broad-host-range cosmids for construction of high quality metagenomic libraries. J Microbiol Methods. 2014;99C:27-34

51. Troeschel SC, Drepper T, Leggewie C, Streit WR, Jaeger K-E. Novel tools for the functional expression of metagenomic DNA. Methods Mol Biol. 2010:668:117-39.

52. Uchiyama T, Miyazaki K. Functional metagenomics for enzyme discovery: challenges to efficient screening. Curr Opin Biotechnol. 2009;20:616-22.

53. Engel K, Pinnell L, Cheng J, Charles TC, Neufeld JD. Nonlinear electrophoresis for purification of soil DNA for metagenomics.J Microbiol Methods. 2012;88:35-40.

54. Kent WJ. BLAT-the BLAST-like alignment tool. Genome Res. 2002;12:656-64. 
55. Nawrocki EP, Eddy SR. Infernal 1.1: 100-fold faster RNA homology searches. Bioinformatics. 2013;29:2933-5.

56. Wang Q, Garrity GM, Tiedje JM, Cole JR. Naive Bayesian classifier for rapid assignment of rRNA sequences into the new bacterial taxonomy. Appl Environ Microbiol. 2007;73:5261-7.

57. Huson DH, Mitra S, Ruscheweyh H-J, Weber N, Schuster SC. Integrative analysis of environmental sequences using MEGAN4. Genome Res. 2011;21:1552-60.

58. Canadian MetaMicrobiome Library Publication Data. http://www.cm2bl.org/ data. Accessed 26 November 2014

\section{Submit your next manuscript to BioMed Central} and take full advantage of:

- Convenient online submission

- Thorough peer review

- No space constraints or color figure charges

- Immediate publication on acceptance

- Inclusion in PubMed, CAS, Scopus and Google Scholar

- Research which is freely available for redistribution 